PROCEEDINGS OF THE

AMERICAN MATHEMATICAL SOCIETY

Volume 127, Number 8, Pages 2469-2473

S 0002-9939(99)04780-2

Article electronically published on April 8, 1999

\title{
SOME REMARKS ON METRIC SPACES WHOSE PRODUCT WITH EVERY LINDELÖF SPACE IS LINDELÖF
}

\author{
K. ALSTER
}

(Communicated by Alan Dow)

\begin{abstract}
Let us assume that Martin's Axiom holds. We prove that if $X$ is a metrizable space whose product with every Lindelöf space is Lindelöf, then for every metric $d$ on $X$, consistent with the topology of $X,(X, d)$ is a countable union of totally bounded subsets.
\end{abstract}

E. Michael proved that under the Continuum Hypothesis, abbreviated $(\mathrm{CH})$, if $X$ is a metric space whose product with every Lindelöf space is Lindelöf, then $X$ is $\sigma$-compact. It is an open question whether $(\mathrm{CH})$ can be relaxed to Martin's Axiom, abbreviated (MA). The aim of this note is to prove the result mentioned in the abstract.

The topological terminology follows [E], and we have adopted set-theoretical terminology from [K2]. In this note, $N$ stands for the set of natural numbers and $P$ for the space of the irrational numbers. The irrational numbers will be viewed as sequences of natural numbers. The symbol $\mathfrak{c}$ will denote the initial ordinal number of the cardinality continuum. Other ordinal numbers will be denoted by Greek letters. If $x=(x(n))_{n=1}^{\infty}$ and $y=(y(n))_{n=1}^{\infty}$ are irrational numbers, we say that $x<_{*} y, x \leq_{*} y$ or $x=_{*} y$ if for all but finitely many $n x(n)<y(n), x(n) \leq y(n)$ or $x(n)=y(n)$ respectively. If $A$ is a subset of a metric space $(X, d)$, then the symbol $\operatorname{diam}(A)<\epsilon$ means that the distance between any two points of $A$ is less than $\epsilon$.

Theorem (MA). If $X$ is a metrizable space whose product with every Lindelöf space is Lindelöf, then the following conditions hold:

a) For every metric $d$ on $X$, consistent with the topology of $X,(X, d)$ is a countable union of totally bounded subsets.

b) Every closed cover of $X$ of cardinality less than continuum has a countable subcover.

c) If $X$ is an analytic space then $X$ is $\sigma$-compact.

Proof of a). Let us first assume that $(X, d)$ is a zero-dimensional space. Note that since $X$ is separable, $X=Z \cup A$, where $A$ is $\sigma$-compact and $Z$ is closed and not locally compact at any point. Without loss of generality we can assume that $X=Z$.

Received by the editors November 12, 1996 and, in revised form, October 31, 1997.

1991 Mathematics Subject Classification. Primary 54B10, 54D20.

Key words and phrases. Metric spaces, product, Lindelöf spaces.

(C)1999 American Mathematical Society 
There is a sequence $\left(\mathcal{U}_{i}\right)_{i=1}^{\infty}$, where $\mathcal{U}_{i}=\left\{U_{p}: p \in N^{i}\right\}$, of open covers of $X$ such that

1) $\mathcal{U}_{i}$ consists of pairwise disjoint non-empty sets,

2) if $U_{p} \in \mathcal{U}_{i}$ then $\operatorname{diam}\left(U_{p}\right)<\frac{1}{i}$,

3) if $p \in N^{i}$ and $q \in N^{i+1}$ and $q$ extends $p$, then $U_{q} \subset U_{p}$.

Let us define a continuous function $g$ of $X$ to $P$ by the following formulas: $g(x)=p_{x}$, where $p_{x} \in P$ is such that $\{x\}=\bigcap_{n=1}^{\infty} U_{p_{x} \mid n}, p_{x}=\left(p_{x}(n)\right)_{n=1}^{\infty}$ and $p_{x} \mid n=\left(p_{x}(1), \ldots, p_{x}(n)\right)$. It is obvious that $g$ is well defined and that it is a continuous function.

Let $\left\{x_{\alpha}: \alpha<\mathfrak{c}\right\}$ be a $\mathfrak{c}$-scale in $P$, which exists under (MA) (see [K2], exercise 8, p. 87).

Claim. There is $\alpha<\mathfrak{c}$ such that

$$
g(X) \subset\left\{p \in P: p \leq_{*} x_{\alpha}\right\}=P_{\alpha}
$$

Assume that the claim holds. Observe that if $\left\{p \in P: p={ }_{*} x_{\alpha}\right\}=\left\{p_{n}: n \in N\right\}$ and $Z_{n}=\left\{p \in P: p \leq p_{n}\right\}$, then $g^{-1}\left(Z_{n}\right)=X_{n}$ is totally bounded for $n \in N$. Indeed if $\epsilon>0, \frac{1}{i}<\epsilon$, and $T=\left\{t \in N^{i}: t \leq p_{n} \mid i\right.$ and $\left.U_{t} \cap X_{n} \neq \varnothing\right\}$, then let $x_{t}$ be a point of $U_{t} \cap X_{n}$ for $t \in T$. Observe that the union of balls with centers at $x_{t}$, for $t \in T$, and radius $\epsilon$ covers $X_{n}$; and this means that $X_{n}$ is totally bounded.

Proof of Claim. Suppose that

4) $g(X) \backslash P_{\alpha} \neq \varnothing$ for $\alpha<\mathfrak{c}$.

Let $Y$ be a metric compactification of $P$ and put $P_{\mathfrak{c}}=Y$. The topology on $P_{\mathfrak{c}}$ is generated by the sets of the form $P_{0} \cap H$ or $\left(P_{\alpha_{2}} \backslash P_{\alpha_{1}}\right) \cap H$, where $0 \leq \alpha_{1}<\alpha_{2} \leq \mathfrak{c}$ and $H$ is open in $Y$. In [A1] it was proved that $P_{\mathfrak{c}}$ is Lindelöf. Observe that $K=\{(y, y): y \in g(X)\}$ is a closed subset of $g(X) \times P_{\mathfrak{c}}$; from 4) it follows that the family $\left\{\left(g(X) \times P_{\alpha}\right) \cap K: \alpha<\mathfrak{c}\right\}$ covers $K$ and does not have a countable subcover, contradicting the Lindelöf property of $g(X) \times P_{\mathrm{c}}$.

Let $X$ be an arbitrary metric space whose product with every Lindelöf space is Lindelöf. There exist a zero-dimensional metric and separable space $Y$ and a perfect continuous function $f$ of $Y$ onto $X$. Since a perfect preimage of a Lindelöf space is Lindelöf, we conclude that for every Lindelöf space $Z$ the product $Y \times Z$ is Lindelöf. Let $d_{1}$ and $d$ be metrics on $Y$ and $X$ respectively. Let us define a new metric $d_{2}$ on $Y$ by Then

5) $d_{2}\left(y_{1}, y_{2}\right)=d_{1}\left(y_{1}, y_{2}\right)+d\left(f\left(y_{1}\right), f\left(y_{2}\right)\right)$.

$$
\left(Y, d_{2}\right)=\bigcup_{n=1}^{\infty} Y_{n}
$$

where $Y_{n}$ is totally bounded. As $d_{2}\left(y_{1}, y_{2}\right) \geq d\left(f\left(y_{1}\right), f\left(y_{2}\right)\right)$, we conclude that $f\left(Y_{n}\right)$ is totally bounded in $(X, d)$.

Proof of b). Let $\left\{X_{\alpha}: \alpha<\lambda\right\}$ be a closed cover of $X$ with $\lambda<\mathfrak{c}$ which does not have a countable subcover. Let $\left(Y, \tau^{\prime}\right)$ be a metric compactification of $X$ and $\tau$ a new topology on $Y$ generated by $\left\{X_{\alpha}: \alpha<\lambda\right\}$ and $\tau^{\prime}$. Put $Z=(Y, \tau)$. By (MA) and the fact that $Z \backslash X$ is a Lindelöf subset of $Z$, we infer that $Z$ is Lindelöf. The product $X \times Z$ is not Lindelöf, because $K=\{(x, x): x \in X\}$ is a closed subset of $X \times Z$ and $\left\{\left(X \times X_{\alpha}\right) \cap K: \alpha<\lambda\right\}$ is an open cover of $K$ without a countable subcover. 
Proof of c). By the Hurewicz theorem, (see [K1], theorem 21.18), if $X$ is not $\sigma$ compact then $X$ contains a closed subset homeomorphic to the irrationals. By the example from [A1] there is a Lindelöf space $Y$ such that $X \times Y$ is not Lindelöf.

Question 1 (MA). Let $X$ be a metrizable separable space such that for every metric $d$ on $X$, consistent with the topology of $X,(X, d)$ is a countable union of totally bounded subsets. Is it true that $X$ is a union of less than continuum compact sets?

We do not know the answer to this question even for $(\mathrm{CH})$. In this case we ask if $X$ is $\sigma$-compact.

Remark 2. Observe that the following conditions are equivalent for a metrizable separable space $X$ :

(i) For every metric $d$ on $X$, consistent with the topology of $X,(X, d)$ is a countable union of totally bounded subsets,

(ii) For every complete metric space $Y$ containing $X$ there is a $\sigma$-compact set $Z$ such that $X \subset Z \subset Y$.

Proof. (i) $\rightarrow$ (ii). If $X \subset(Y, d)$, where $(Y, d)$ is a complete metric space, then by (i) $(X, d \mid X \times X)=\bigcup_{n=1}^{\infty} X_{n}$, where $X_{n}$ is totally bounded, and $\bar{X}_{n}^{Y}$ is compact as a complete totally bounded metric space.

(ii) $\rightarrow$ (i). There is a complete separable metric space $\left(Y, d^{\prime}\right)$ such that $(X, d) \subset$ $\left(Y, d^{\prime}\right)$ and $d^{\prime} \mid X \times X=d$ (see [E], Theorem 4.3.14). By (ii) there is $Z$ such that $X \subset Z=\bigcup_{n=1}^{\infty} Z_{n} \subset Y$, where $Z_{n}$ is compact. Then $X_{n}=Z_{n} \cap X$ is totally bounded.

Another proof of this implication follows from the fact that (ii) implies that there is $a \in P$ such that $g(X) \subset\{p \in P: p \leq a\}$, where $g$ is taken from the proof of part a) of the theorem.

Remark 3. One can also prove the theorem using Remark 2, but in this case the reasoning is not so constructive as in the original proof (cf. [A2], Remark 16).

Question 4. Is (MA) essential in the theorem?

Question 5. Is it consistent with ZFC to assume that there is a metric space $(X, d)$ which is not a countable union of totally bounded subsets and whose product with every Lindelöf space is Lindelöf?

Question 5 is related to a well known problem:

Question 6. Is it consistent with ZFC to assume that for every Lindelöf space $Y$ the product $Y \times P$ is Lindelöf?

Let us finish with the following:

Proposition (MA). If $X$ is a metrizable space whose product with every Lindelöf space is Lindelöf, then the following condition holds:

There is an increasing sequence $\left\{A_{\alpha}: \alpha<\lambda\right\}$ of $\sigma$-compact subsets in $X$, where $\lambda<\mathfrak{c}$, which cannot be extended, which means that there is no $\sigma$-compact set $Z$ in $X$ containing $\bigcup\left\{A_{\alpha}: \alpha<\lambda\right.$ as a proper subset.

Moreover, if in addition $X$ is not $\sigma$-compact, then there is a compact set $C$ in $X$ such that $\left\{\alpha<\lambda: A_{\alpha}^{\prime} \cap C \neq \varnothing\right\}$ is cofinal with $\lambda$, where $A_{\alpha}^{\prime}=A_{\alpha} \backslash \bigcup\left\{A_{\beta}: \beta<\alpha\right\}$. 
Proof. Suppose not, and let $\left\{C_{\alpha}: \alpha<\mathfrak{c}\right\}$ be the family of all compact subsets of $X$. Put $A_{0}=C_{0}$. If $A_{\alpha}$ has been defined for $\alpha<\beta$, then by the assumption there is a $\sigma$-compact set $Z$ such that the union of $\left\{A_{\alpha}: \alpha<\beta\right\}$ is a proper subset of $Z$. Put $A_{\beta}=Z \cup C_{\beta} \cup\left\{x_{\beta}\right\}$, where $x_{\beta}$ is an arbitrary point of $X \backslash \bigcup\left\{A_{\alpha}: \alpha<\beta\right\}$.

From the construction it follows that

$$
\bigcup\left\{C_{\alpha^{\prime}}: \alpha^{\prime}<\alpha\right\} \subset \bigcup\left\{A_{\alpha^{\prime}}: \alpha^{\prime}<\alpha\right\} \text { for } \alpha<\mathfrak{c} .
$$

Let $\left(Y^{\prime}, \tau_{1}\right)$ be a metrizable compactification of $X$. Then put $A_{\mathfrak{c}}=Y^{\prime}$ and $Y=\left(Y^{\prime}, \tau_{2}\right)$, where $\tau_{2}$ is generated by $\tau_{1} \cup\left\{A_{\alpha} \backslash A_{\alpha^{\prime}}: \alpha^{\prime}<\alpha \leq \mathfrak{c}\right\} \cup\left\{A_{0}\right\}$.

We shall show that $Y$ is a Lindelöf space and $X \times Y$ is not. Observe that $Y \backslash X$ is a Lindelöf space as a subspace of $\left(Y^{\prime}, \tau_{1}\right)$. Let $U$ be an open subset of $Y$ containing $Y \backslash X$. Then there is a $\sigma$-compact set $C$ in $X$ such that $Y \backslash U \subset C$, and by (*) there is $\alpha$ such that $C \subset A_{\alpha}$. To finish the proof of the Lindelöf property of $Y$ it is enough to show that $A_{\alpha}$ is a Lindelöf subspace of $Y$ for all $\alpha$. It is obvious that $A_{0}$ is Lindelöf. Suppose that $A_{\beta}$ is Lindelöf for $\beta<\alpha$. Note that $A_{\alpha}^{\prime}=A_{\alpha} \backslash \bigcup\left\{A_{\beta}: \beta<\alpha\right\}$ is Lindelöf as a subspace of $X$. Let $U$ be an open set in $Y$ containing $A_{\alpha}^{\prime}$. Then there is a $\sigma$-compact set $D$ in $X$ such that $A_{\alpha} \backslash U \subset D \subset \bigcup\left\{A_{\beta}: \beta<\alpha\right\}$. Since (MA) implies that every closed cover of a metrizable compact set of cardinalinality less than continuum has a countable subcover (see [A1], Lemma 2), by the inductive assumption we conclude that $A_{\alpha}$ is a Lindelöf space.

The product $X \times Y$ is not Lindelöf, because $K=\{(x, x): x \in X\}$ is a closed subset of $X \times Y$ and the family $\left\{K \cap\left(X \times A_{\alpha}\right): \alpha<\mathfrak{c}\right\}$ is an open cover of $K$ without countable subcover.

Suppose that $\left\{A_{\alpha}: \alpha<\lambda\right\}$ is an increasing sequence of $\sigma$-compact subsets in $X$, where $\lambda<\mathfrak{c}$, and there is no a compact subset $C$ in $X$ such that $\{\alpha<\lambda$ : $\left.A_{\alpha}^{\prime} \cap C \neq \varnothing\right\}$ is cofinal with $\lambda$, where $A_{\alpha}^{\prime}=A_{\alpha} \backslash \bigcup\left\{A_{\beta}: \beta<\alpha\right\}$. Then, using a similar reasoning as in the first part of the proof, we conclude that $T=\bigcup\left\{A_{\alpha}\right.$ : $\alpha<\lambda\} \cup Y \backslash X$ is a Lindelöf subspace of $Y$ and $X \times T$ is not Lindelöf.

Remark 7 . One can strengthen the second part of the proposition by showing that if $Z$ is a $\sigma$-compact set then there is a compact set $C$ in $X$ such that $\{\alpha<\lambda$ : $\left.(C \backslash Z) \cap A_{\alpha}^{\prime} \neq \varnothing\right\}$ is cofinal with $\lambda$.

Question 8 (MA). Let $\lambda^{\prime}$ be an ordinal number less than continuum. Is it possible to relax the phrase " $\sigma$-compact", in the formulation of the proposition, to "union of $\lambda^{\prime}$ compact sets"?

Added in proof. J. Chaber pointed out to me that W. Hurewicz (On sequences of continuous functions, Fund. Math. 9(1927), 193-204) proved that a metric space $M$ is a Hurewicz space if and only if every sequence $\left(f_{n}\right)$ of continuous real valued functions on $M$ is bounded, i.e. there is a sequence $\left(r_{n}\right)$ of real numbers such that for all but finitely many $n$ the range of $f_{n}$ is bounded by $r_{n}$.

Recently R. Pol has answered Question 1 in the negative way.

\section{REFERENCES}

[A1] K. Alster, On the product of a Lindelöf space with the space of irrationals, Proc. Amer. Math. Soc. 110 (1990), 543-547. MR 90m:54012

[A2] K. Alster, Some remarks concerning the Lindelöf property of the product of a Lindelöf space with the irrationals, Topology and its Applications 44 (1992), 19-25. MR 93g:54013 
[E] R. Engelking, General Topology, Heldermann Verlag, Berlin, 1989. MR 91c:54001

[K1] A.S. Kechris, Classical Descriptive Set Theory, Graduate Texts in Math., vol. 156, SpringerVerlag, Berlin, 1995. MR 96e:03057

[K2] K. Kunen, Set theory: An introduction to independence proofs, Stud. Logic Foundations Math., vol. 102, North-Holland, Amsterdam, 1980. MR 82f:03001

Institute of Mathematics, Polish Academy of Sciences, 00-950 Warsaw, Poland

E-mail address: kalster@impan.gov.pl 